\title{
Allometric Scaling of Long-Distance Seed Dispersal by Migratory Birds
}

\author{
Duarte S. Viana, ${ }^{1, \star}$ Luis Santamaría, ${ }^{2}$ Thomas C. Michot, ${ }^{3}$ and Jordi Figuerola ${ }^{1}$ \\ 1. Department of Wetland Ecology, Estación Biológica de Doñana (EBD-CSIC), Apartado de Correos 1056, E-41080 Sevilla, Spain; \\ 2. Mediterranean Institute for Advanced Studies (IMEDEA, CSIC-UIB), Miquel Marqués 21, E07190 Esporles, Mallorca, Balearic \\ Islands, Spain; 3. Institute for Coastal Ecology and Engineering, University of Louisiana at Lafayette, Louisiana
}

Submitted July 13, 2012; Accepted January 14, 2013; Electronically published March 15, 2013

Online enhancements: appendixes. Dryad data: http://dx.doi.org/10.5061/dryad.619gd.

\begin{abstract}
AвSTRACT: Migratory birds are often suggested to be important vectors for long-distance dispersal (LDD) of plant and animal propagules. The scale of such dispersal events (hundreds to thousands of kilometers) can influence landscape-level biological processes and species distributions. However, the few vector species studied and the lack of proper integration of their migratory movement in models of LDD has precluded the study of their potential as long-distance biotic dispersers. By means of a mechanistic model parameterized with empirical data, we first investigated the properties of seed dispersal curves generated by migratory birds and then analyzed the effect of bird size on model parameters and consequent seed dispersal patterns. Seed dispersal curves showed in most cases large and heavy tails, resulting in relatively frequent LDD (up to $3.5 \%$ of dispersal distances longer than $100 \mathrm{~km}$ ). Bird size mediated trade-offs between bird movement and seed retention time that, in turn, determined seed dispersal patterns and the potential of each bird species as an LDD vector. Our modeling framework builds on a mechanistic understanding of seed dispersal by migratory birds and may thus be a useful tool to estimate the scale and frequency of bird-mediated, large-scale transport of native, invasive, and pathogenic organisms.
\end{abstract}

Keywords: long-distance dispersal, migratory birds, seed dispersal, body size, endozoochory, mechanistic model.

\section{Introduction}

Long-distance dispersal (LDD) of animal-dispersed propagules arises from relatively infrequent large-scale movements by the dispersers, which often cause dispersal curves (describing the frequency distribution of dispersal distances) to be fat-tailed (Nathan et al. 2008). Notwithstanding its low frequency, LDD can have important ecological consequences. It might link habitat patches in fragmented landscapes, where stepping-stone dispersal is constrained (Levey et al. 2008), and influence the dispersal rates of individuals

\footnotetext{
* Corresponding author; e-mail: dviana@ebd.csic.es.
}

Am. Nat. 2013. Vol. 181, pp. 649-662. (c) 2013 by The University of Chicago. 0003-0147/2013/18105-53974\$15.00. All rights reserved. DOI: $10.1086 / 670025$ and species (Higgins and Richardson 1999), thus affecting the regional dynamics and structure of populations and communities (Levine and Murrell 2003; Bohrer et al. 2005). When LDD occurs at even larger scales, it might lead to range expansion or shifts (including invasions), influencing biogeographical and evolutionary processes. Furthermore, LDD might be a crucial mechanism for the survival of species or populations currently facing habitat fragmentation and climate change (Trakhtenbrot et al. 2005).

Migratory animals, especially birds, are often suggested to be responsible for the biogeographic patterns of plant and invertebrate species that rely on them for propagule dispersal. Infrequent propagule LDD events produced by large-scale bird movements might explain rapid range expansions following glaciations (e.g., Clark 1998), the colonization of isolated islands (Gillespie et al. 2012), and the existence of certain biogeographic disjunctions (e.g., the bipolar distribution of crowberries; Popp et al. 2011). Unfortunately, the low frequency and presumed unpredictability of LDD have precluded the study of this process in the field. Virtually nothing is known about propagule dispersal kernels produced by migratory animals. Here, we take advantage of well-documented migratory movements of one group of birds (waterbirds; a term used hereafter to designate shorebirds and waterfowl species, which are Charadriiformes and Anatidae, respectively) to study the mechanisms behind the LDD of several aquatic plant species whose seeds are regularly ingested by them. Despite the fragmented distribution of inland bodies of water across continents, aquatic angiosperms have larger ranges than their terrestrial counterparts (Santamaría 2002), suggesting that LDD may be an important process maintaining connectivity between isolated populations of vectored species.

Waterbirds are known to ingest large numbers of propagules, move over long distances, and deposit propagules into suitable habitat (wetlands; Figuerola and Green 2002; Figuerola et al. 2003; Charalambidou and Santamaría 
2005; Brochet et al. 2009b). Furthermore, they might be the only vectors capable of dispersing propagules over regional scales in many aquatic ecosystems. Previous estimates suggest that the seeds and eggs of many aquatic plants and invertebrates can be dispersed over hundreds of kilometers (Charalambidou et al. 2003a; Soons et al. 2008), on the basis of the passage times of seeds or eggs fed experimentally to captive birds. These estimates are in close agreement with the distribution of most vectored species (i.e., those for which viable propagules are regularly found in waterbird feces), as they tend to occur throughout the migration ranges of their respective vectors (Brochet et al. 2009b). Indeed, waterbird movements were found to explain, across a continental scale, a significant fraction of the gene flow among populations of three vectored species of aquatic invertebrates (Figuerola et al. 2005).

Although migratory birds cover extremely large distances during migration, propagules carried in their guts might not be retained there long enough to reach suitable habitat. Instead, ingested propagules could be defecated either before departure or during flight (thus landing, in all likelihood, in unsuitable habitat). Therefore, investigating whether propagule gut retention time (GRT) scales to the time required to complete a given migratory movement (and, consequently, the distance covered by such movement) is crucial to assess the probability of LDD events.

Given the methodological difficulties involved in the study of LDD (a low-frequency event taking place over large geographic scales), mechanistic models have become a widely used tool to investigate its magnitude and patterns. The basic approach for estimating propagule dispersal distances is to combine the vector movement patterns with the GRT of ingested propagules. However, the previous approach to estimate the dispersal distance of propagules ingested by migratory birds (estimated only for waterbirds to date) was to multiply their GRT by bird flight speed (Charalambidou et al. 2003a; Soons et al. 2008), ignoring, for example, the actual distances traveled by the bird. This approach overestimates propagule dispersal distance because it assumes that birds (1) depart immediately after propagule ingestion, (2) move linearly and at constant speed, and (3) land immediately before propagule deposition. In reality, these conditions should be rarely met because birds (1) often take some time to depart, waiting for optimal weather and/or physiological conditions to be met; (2) adjust their movement direction en route depending on, for example, landscape features; and (3) decide to land after a given time independently of the GRT of propagules, for instance, depending on energetic constraints. This approach is thus suited to estimate maximum rather than realized dispersal distances, as shown in Viana et al. (2012). Moreover, because propagule dispersal distances have been recently shown to be affected by the migratory strategies of the dispersers (i.e., the decisions taken by an individual or species, which are reflected in the frequency and distance of migratory movements; Viana et al. 2012), interspecific variation in their migratory movements should be included in mechanistic models of propagule dispersal.

In addition, the development of mechanistic models of zoochorous dispersal has been constrained to date by our limited understanding of the disperser characteristics that influence both their movement behavior and the seeds' retention time (Cousens et al. 2010). For example, body size is known to influence the movement of flying animals (especially migratory movement; e.g., Hein et al. 2012) as well as the gut passage rate of food items (e.g., Karasov 1990), and it may therefore be an important determinant of migratory movement and propagule GRT.

Given the lack of integration of the birds' actual movement patterns and characteristics in estimates of longdistance propagule dispersal, we developed a mechanistic model, parameterized with empirical information obtained from various bird species, to provide a general framework to understand and estimate propagule dispersal by migratory birds. Using this mechanistic framework, we simulated the dispersal of seeds by migratory waterbirds to (1) estimate seed dispersal patterns generated by the migratory movements, (2) analyze the relative importance of model parameters related to the migratory behavior of dispersers and to the GRT of seeds for the resulting seed dispersal patterns, and (3) investigate how bird body size scales to estimated model parameters and, through them, to estimated seed dispersal patterns.

\section{Methods}

\section{Modeling Framework}

We designed a rule-based stochastic model to simulate the dispersal of seeds ingested by birds during the migration season. The model allowed us to take into account the stochasticity inherent to LDD processes (as noted in $\mathrm{Na}$ than et al. 2003), which arises (in our model) from the random association between the GRT of each ingested seed and the bird's movement (or lack thereof) during that time (see below). The simulation also allowed us to easily estimate the frequency of transported seeds and the frequency of LDD events, which are important to estimate the rate at which a given plant (or other passively dispersed organism) might be dispersed. Our modeling approach (simulation) represents a convenient method because the combination of equations parameterized with the data available for this study, used to represent the frequency distributions of the concatenated processes determining 


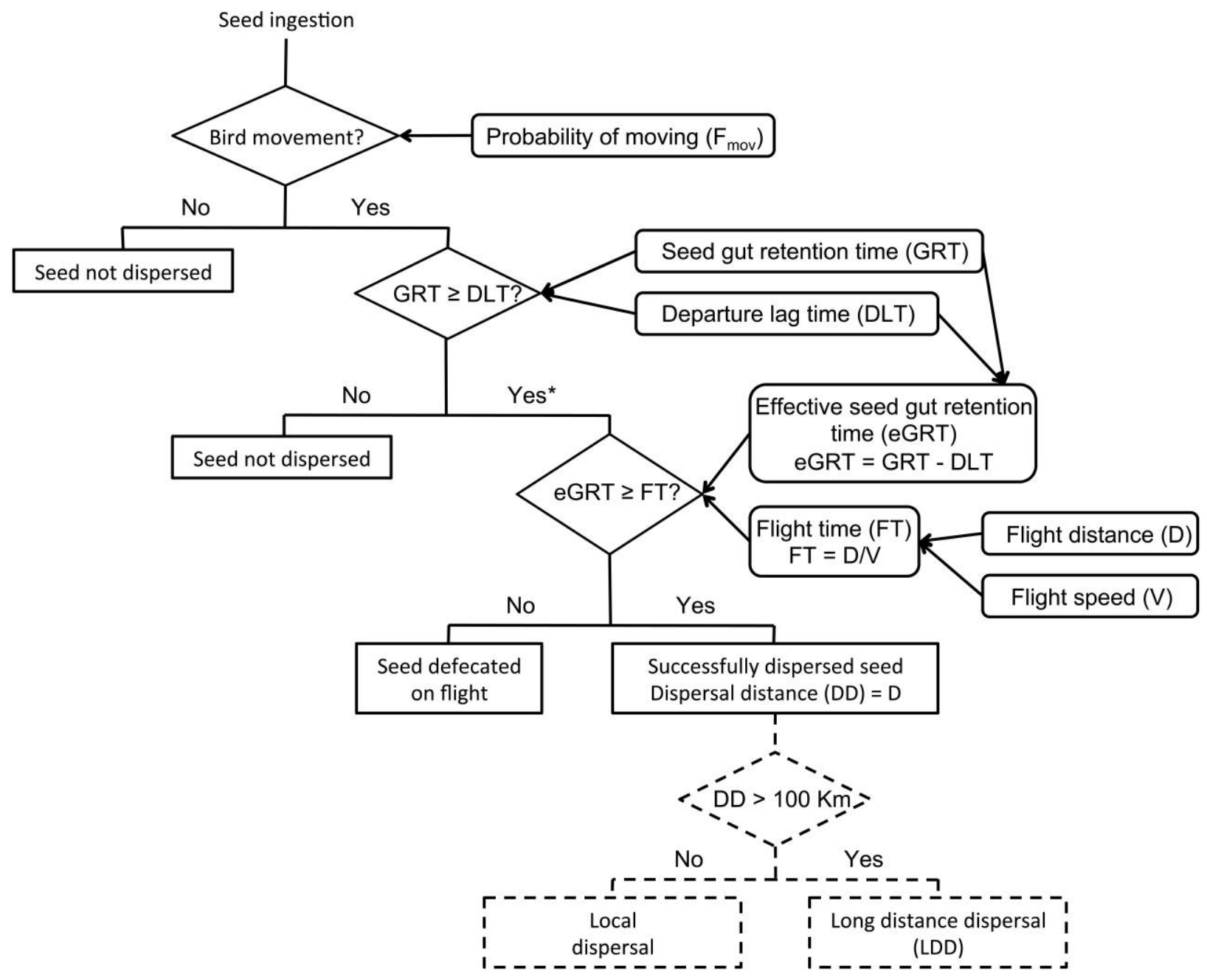

Figure 1: Flow diagram of the conceptual model of seed dispersal by migratory birds. Diamonds represent the rules of the dispersal process, rectangular boxes represent model processes, and rounded rectangular boxes represent input variables. Because the definition of longdistance dispersal (dispersal distances longer than $100 \mathrm{~km}$ ) is specific for these dispersers (waterbirds) but the rest of the model might be generalized (to other migratory bird dispersers), we represented this process with dashed lines. The asterisk indicates seed transportation.

the output variables (seed dispersal), is not amenable to analytical solution (see app. A, available online).

Model flow (fig. 1) was designed to reproduce the bird's behavior after seed ingestion. First, after ingesting a seed the bird could stay immobile until seed defecation (in which case the dispersal distance is 0 , i.e., no dispersal occurs) or move according to the probability of moving $\left(F_{\text {mov }}\right)$. If the bird moves but the GRT of the seed is shorter than the time the bird takes to depart (departure lag time [DLT]), the seed is defecated before departure and therefore is not dispersed. If movement starts before seed defecation (in which case the DLT is subtracted from the GRT, and the remaining GRT is called effective GRT [eGRT]), the bird can stop either after seed defecation (flight time longer than the eGRT, in which case the seed is defecated, while flying, into unsuitable habitat and no dispersal occurs) or before (flight time shorter than the eGRT, and the seed is dispersed at the distance equal to the flight distance; see also Viana et al. 2012). Within the GRT, each bird could either move locally or make a migratory flight, with probabilities and distances tailored to the movement pattern of each bird species (see below). In this study, we considered that LDD events are those produced by migratory movements (defined as those longer than $100 \mathrm{~km}$ ). Figure 1 represents the flow diagram of the conceptual dispersal model (each simulation comprised 1 million iterations), and table 1 shows the model parameters (described below). 
Table 1: Summary information on model parameters

\begin{tabular}{llc}
\hline Component, parameter & \multicolumn{1}{c}{ Description } & Range \\
\hline $\begin{array}{l}\text { Bird movement: } \\
F_{\text {mov }}\end{array}$ & Probability of moving & $.03-.08$ \\
DLT & Departure lag time (h) & $.5-6$ \\
$V$ & Flight speed (km/h) & $45-85$ \\
$F_{\text {mig }}$ & Frequency of migratory movements & $.08-.56$ \\
$\mu_{\text {local }}$ & Scale of lognormal (fitted to the distance distribution of local movements) & $1.92-3.33$ \\
$\sigma_{\text {local }}$ & Shape of lognormal (fitted to the distance distribution of local movements) & $.49-1.16$ \\
$\mu_{\text {mig }}$ & Scale of lognormal (fitted to the distance distribution of migratory movements) & $5.23-6.58$ \\
$\sigma_{\text {mig }}$ & Shape of lognormal (fitted to the distance distribution of migratory movements) & $.49-.97$ \\
Seed gut retention time: & & \\
$\mu_{\text {GRT }}$ & Scale of lognormal (fitted to the seed retention time distributions) & -.01 to 2.41 \\
$\sigma_{\text {GRT }}$ & Shape of lognormal (fitted to the seed retention time distributions) & $.11-1.20$ \\
\hline
\end{tabular}

Note: The minimum and maximum values of the parameters were rounded down and up, respectively.

\section{Model Parameterization: Bird Movement}

Probability of Moving $\left(\mathrm{F}_{\text {mov }}\right)$. In birds that move by flapping flight (as is the case for waterbirds and most seed consumers), species-specific ratios between flying time and stopover time range from $1: 14$ to $1: 30$ (Newton 2008). This means that the probability of moving ranges from approximately 0.03 to $0.08\left(F_{\text {mov }}\right)$ during the migration season.

Departure Lag Time (DLT). DLT represents the time elapsed between propagule ingestion and bird departure. This parameter varied according to three behaviors: (1) DLT decreases exponentially, which means that the moment of departure often takes place soon after propagule ingestion (Clausen et al. 2002); (2) DLT increases exponentially, which means that waterbirds tend not to feed before departure (Zwarts et al. 1990); and (3) DLT is uniformly distributed, which means that birds depart randomly, independently of the time of feeding (Owen 1968). Because data on DLT are not available in the literature (we are aware only of an example with swans, in Clausen et al. 2002), we chose an arbitrary exponent value $(-1.2$ and 1.2 for behaviors 1 and 2, respectively) to model these behaviors and focused the sensitivity analysis on investigating the effect of the different behaviors on the dispersal patterns rather than the effect of variation in specific parameter values.

Movement Distance. Waterbird (waterfowl and shorebird) movement was analyzed using banding data from EURING (European Union for Bird Banding) and the Bird Banding Laboratory (US Geological Survey). All banding and recovery records were submitted to several accuracy filters because of inherent inaccuracies in the data collection (often band recoveries for which the place and date were inexactly reported by hunters). Maximum error margins were 1 day for dates and $10 \mathrm{~km}$ for geographical position (beyond these thresholds, all data were excluded). Only recoveries of dead birds, mainly shot, were used, because dead-bird recoveries (which are mainly reported by hunters) are considered to be more representative of actual bird distribution than those reported by banders (Wernham et al. 2002). Only data from the autumn migration season (from July to November) were included, as sample sizes (no. of recoveries) for the spring migration season were too low.

The occurrence of a successful dispersal event depended on the comparison of the flight time with the seed retention time (see "Modeling Framework"). However, the flight time is not the direct measure of the time elapsed between banding and recovery, as the latter include immobility periods (e.g., stopover periods) and possibly more than a single migratory movement. Because the duration of immobility periods and single movements (occurring within banding and recovery) cannot be discriminated in our data set, we limited the time elapsed between banding and recovery to the minimum stopover time during migration (6 days for waterfowl and 3 days for shorebirds). These time windows were chosen on the basis of our literature search (Miller et al. 2005; Lehnen and Krementz 2007; O'Neal et al. 2012), which indicated that they are unlikely to include more than single migratory movements. Hence, the flight time (FT) corresponding to the distance of that single migratory movement can be calculated by dividing the flight distance $(D$; distance from banding to recovery) by the flight speed ( $V$; see "Modeling Framework"). If within the chosen time window the birds could make more than one migratory movement, then the distance traveled between banding and recovery would increase with the corresponding time (because the likelihood of performing a second movement would increase with time). The lack of significant correlations between distance and time up to the minimum stopover time mentioned above suggests that our assumption is correct (see 
app. B, table B1, available online; data available in Dryad: http://dx.doi.org/10.5061/dryad.619gd).

To model bird movement distances, we fitted a mixture of two lognormal distributions to the distribution of movement distances of each waterbird species, so that local and migratory movements could be simultaneously characterized by independent parameters. Migratory movements were considered those longer than $100 \mathrm{~km}$ because according to the most exhaustive survey of waterfowl movements by means of satellite telemetry (to our knowledge; 228 individuals of 19 species; Gaidet et al. 2010) movements longer than $100 \mathrm{~km}$ can be considered migratory and because local movements (in winter) are usually shorter than $50 \mathrm{~km}$. Moreover, fat-tailed single distributions (such as single lognormal, Pareto, gamma, and Weibull) failed to represent long-distance movements (the tail of the distribution; data not shown), whereas the proposed mixture distribution finely represented the tail of the distribution (see app. B, fig. B1). The 100-km cutoff is therefore supported by the data and also allowed us to have two independent descriptors of migratory distance (which were used for allometric relations with bird mass).

The mixture distribution was scaled up using mixture weights based on the observed frequencies of local $(\leq 100$ $\mathrm{km})$ and migratory $(>100 \mathrm{~km})$ movements $\left(F_{\text {local }}+\right.$ $\left.F_{\text {mig }}=1\right)$. The probability density function (PDF) of the final (mixture) distribution is given by

$$
\begin{aligned}
f(x)= & \left(1-F_{\text {mig }}\right) p\left(x \mid \mu_{\text {local }}, \sigma_{\text {local }}\right) \\
& +F_{\text {mig }} p\left(x \mid \mu_{\text {mig }}, \sigma_{\text {mig }}\right),
\end{aligned}
$$

where $p(x)$ is the PDF of a 2-parameter lognormal distribution,

$$
p(x \mid \mu, \sigma)=\frac{1}{\sqrt{2 \pi} \sigma x} \exp -\left(\frac{(\ln x-\mu)^{2}}{2 \sigma^{2}}\right),
$$

and $\mu$ and $\sigma$ are the scale and shape parameters, respectively. The distributions were fitted to data by maximum likelihood estimation using the package "fitdistrplus" and further handled (assemblage of mixture distribution, random generation of values, and truncation of distributions) using the package "distr" of R software (R Development Core Team 2009). All fitted distributions were truncated at 3,000 $\mathrm{km}$ because satellite data showed that waterfowl make single migratory displacements of up to $2,900 \mathrm{~km}$ (Gaidet et al. 2010). Table B2 (app. B) shows the goodnessof-fit test results for all 13 species present in either Europe or North America (see also fig. B1).

For the sensitivity analysis (see below), the parameters of the lognormal distribution (scale, $\mu$, and shape, $\sigma$ ) corresponding to local distances $\left(\mu_{\text {local }}\right.$ and $\left.\sigma_{\text {local }}\right)$ were kept constant in all simulations, since we did not aim at quantifying local dispersal distances (only the frequency of local vs. longdistance dispersal; see "Model Outputs"). The range of variation for the parameters defining the migratory movement-the frequency of migratory (vs. local) movements $\left(F_{\text {mig }}\right)$ and the scale $\left(\mu_{\text {mig }}\right)$ and shape $\left(\sigma_{\text {mig }}\right)$ of migratory distances-was tailored to reflect the range of interspecific variation present in our migratory movement data set.

Flight Speed (V). We used airspeed (i.e., the speed relative to the air mass, as opposed to ground speed, which is the speed relative to the ground and is thus influenced by the wind speed and direction) despite the potential effect that air density and wind might have on the actual ground speed because it allowed us to select the range of values $(45-85 \mathrm{~km} / \mathrm{h})$ based on measures taken directly on freeflying waterfowl and wader species (Welham 1994; Bruderer and Boldt 2001; Alerstam et al. 2007). Despite the variation introduced by air density and wind speed, this range of airspeeds probably provides a conservative estimate of the corresponding range of variation in ground speeds among the species used in this study.

\section{Model Parameterization: Seed Retention Time}

We used seed GRT data from three previous studies (Charalambidou et al. 2003b, 2005; Figuerola et al. 2010) in which known numbers of seeds of different aquatic plant species were fed to individuals of seven waterfowl species (see app. B, table B3, for a full list of the species used). To investigate the survival, retention time, and germinability of seeds after gut passage, feces were collected at given time intervals (from 1 to $4 \mathrm{~h}$, depending on the experiment), and the germinability of extracted seeds was tested. Because the exact time of seed defecation was not known (only the time intervals of retrieval from the cage), data were considered to be interval censored (data available in Dryad: http://dx.doi.org/10.5061/dryad $.619 \mathrm{gd})$. Since we were interested in modeling effective seed dispersal, fitted curves were based solely on the retention time of seeds that germinated after gut passage.

The frequency of defecated seeds rapidly increases after ingestion and then decays in a power-law fashion; therefore, we evaluated the fits of three types of parametric distributions: gamma, lognormal, and Weibull distributions. These types of distributions are often used to model seed retention times (e.g., Rawsthorne et al. 2009). The three fittings were compared using the Akaike Information Criterion and the coefficient of determination $\left(R^{2}\right.$, based on the squared coefficient of the correlation between observed and estimated data). Although fits were very similar, the lognormal distribution generally provided slightly better fits (see app. B, table B3, fig. B2). Hence, we decided to fit this distribution (lognormal; PDF is given in eq. [2]) 
to all GRT distributions to enhance parameter and model output comparability. The distributions were fitted to data by maximum likelihood estimation using the package "fitdistrplus." Random generation of values and truncation of distributions were done using the package "distr" of $\mathrm{R}$ software (R Development Core Team 2009). All distributions were truncated at $52 \mathrm{~h}$, which was the maximum seed retention time recorded in the complete data set (see above).

\section{Model Outputs}

Frequency of Effective Dispersal (FED). FED is the proportion of dispersed seeds transported into a different locality (i.e., excluding viable seeds defecated at the site of ingestion or during flight). Note that the denominator of this proportion is the total number of defecated viable seeds (equivalent, in this case, to the number of model iterations); hence, to refer this frequency to the number of ingested seeds, it should be multiplied by the proportion of seeds that survive and remain viable after gut passage, which ranged from $0.5 \%$ to $22 \%$ in the original data sets (Charalambidou et al. 2003b, 2005; Figuerola et al. 2010).

Frequency of $L D D\left(\mathrm{~F}_{L D D}\right)$. LDD events were defined as those produced by migratory movements, and therefore $F_{\mathrm{LDD}}$ is the proportion of effectively dispersed seeds that are transported $>100 \mathrm{~km}$. This cutoff was defined according to the movement pattern showed by waterbirds. Again, the denominator of this proportion is the total number of defecated viable seeds.

Dispersal Distance. We used two components of the seed dispersal curve (the distance distribution of dispersed viable seeds) to characterize seed dispersal distance: the median dispersal distance and the $99 \%$ quantile of the dispersal curve $\left(Q_{99}\right)$.

\section{Sensitivity Analysis}

To estimate the influence of variation in the different parameters on seed dispersal patterns, we carried out a global sensitivity analysis designed to encompass the complete range of variation observed in the empirical data sets (see table 1). The contribution of each parameter to model output was estimated by calculating Sobol's indexes according to the method of Saltelli (Saltelli 2002). This approach can be used regardless of the type of model, as it does not assume linearity of effects. Saltelli's indexes represent the proportion of output variation that is attributed to each parameter, due to both its pure effect (first-order index) and its global effect (including interactions with the other parameters; total index). If the total index is higher than the first-order index, it means that interaction effects are present and contribute to output variation. To ensure that parameter values were drawn from uniform distributions over the parameters' range of values (listed in table 1) and a broad coverage of possible combinations of parameter values, a Latin hypercube sampling technique was applied using the package "lhs" of R software (R Development Core Team 2009). The total number of combinations (and consequently of simulations) was $n(k+$ 2 ), where $n$ is the number of samples $(10,000)$ and $k$ is the number of parameters (eight). The sensitivity analysis was performed using the package "sensitivity" of R software (R Development Core Team 2009).

\section{Effect of Bird Body Mass on Migratory Movement and Seed Retention Time}

To investigate the potential influence of the body mass of various bird species (a surrogate of their body size) on the main determinants of seed dispersal, we fitted linear mixed models to three descriptors of migratory movement patterns $\left(F_{\text {mig }}, \mu_{\text {migg }}\right.$ and $\left.\sigma_{\text {mig }}\right)$ and two descriptors of seed retention time $\left(\mu_{\mathrm{GRT}}\right.$ and $\left.\sigma_{\mathrm{GRT}}\right)$. The scale parameter $(\mu)$ of a lognormal curve is associated with the measures of central tendency, but only the median depends entirely on this parameter (median $=$ exponentiated $\mu$, which in the case of a lognormal distribution is equal to the geometric mean), and the shape parameter $(\sigma)$ is associated with the measures that influence the shape of the distribution (such as the skewness and kurtosis). Mean body mass of each bird species was obtained from Lislevand et al. (2007; male and female values averaged). Body mass was used as the explanatory variable in all models. We included as random effects the continent (Europe and North America) in the models of migratory movement and the experiment (the different origins of the data) and seed species in the models of seed retention time. The significance of body mass was obtained comparing the full model with a submodel without its effect by means of likelihood ratio tests. All models were fitted using the package "Ime4" of R software (R Development Core Team 2009).

\section{Effect of Bird Mass on Seed Dispersal Patterns}

To investigate the effect of bird body mass on seed dispersal patterns, we estimated the model outputs for a range of model parameter values that were made to depend on such body mass. First, we selected a range of variation in bird body mass, chosen to encompass that observed among all bird species used in this study (40-1,260 g, from the dunlin Calidris alpina to the mallard Anas platyrhynchos). Second, we estimated the values of the model parameters that correspond to the different values of bird body mass, based 
A

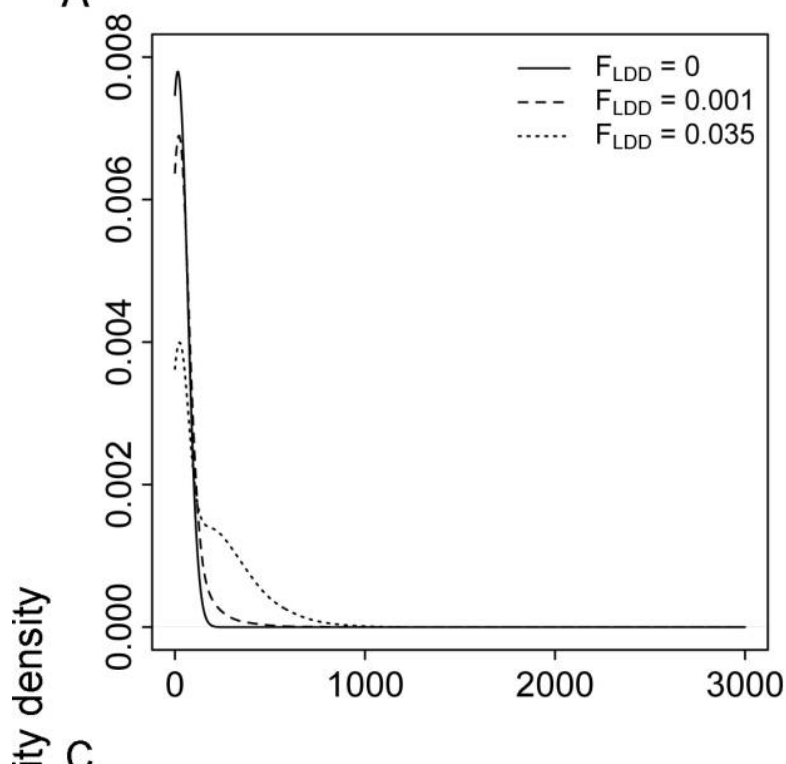

B

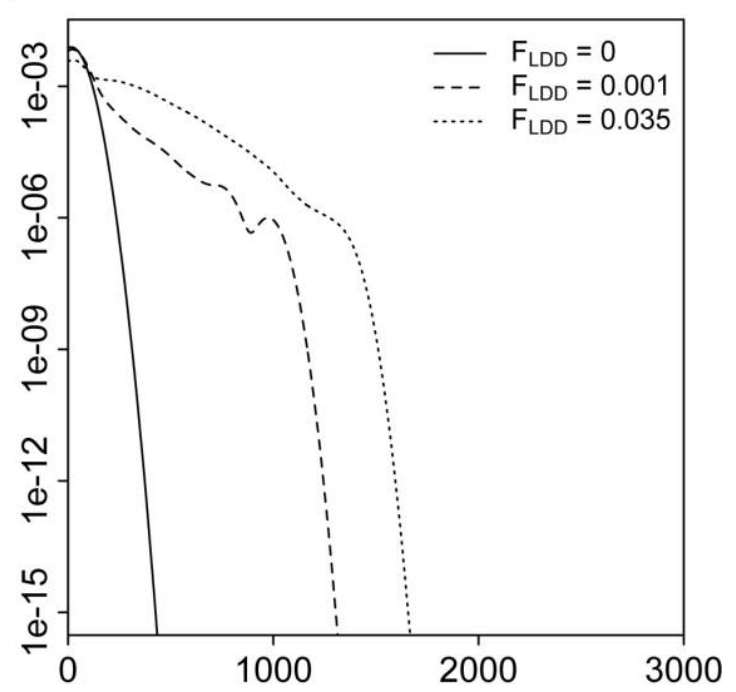

D

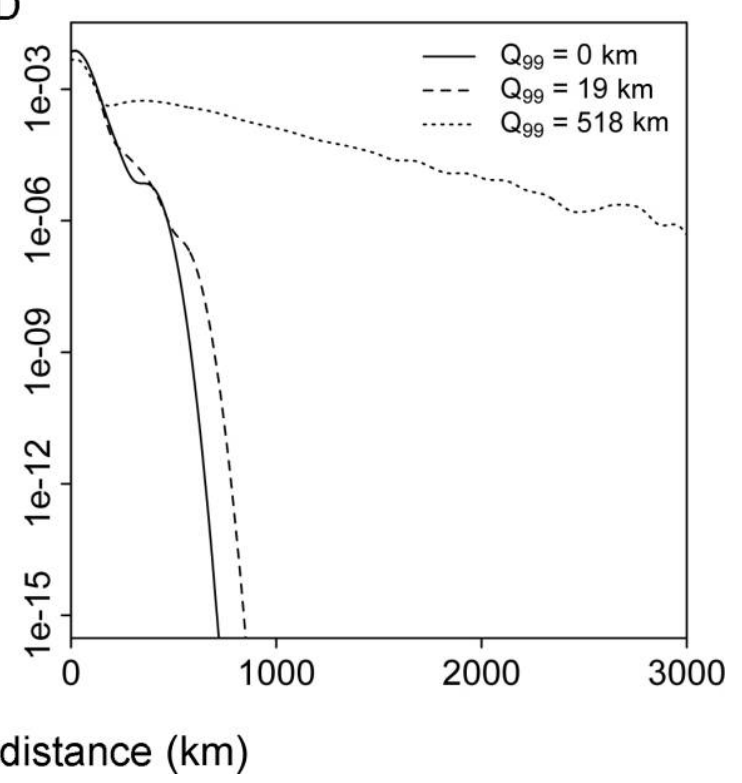

Figure 2: Estimated seed dispersal curves corresponding to the minimum, median, and maximum value of three outputs of the model. Model outputs are the frequency of long-distance dispersal $\left(F_{\mathrm{LDD}} ; A, B ; B\right.$ represents the same as $A$, but the vertical axis is in logarithmic scale to enhance visualization of the curve's tail), median dispersal distance $(C)$, and the $99 \%$ quantile $\left(Q_{99} ; D\right)$. The vertical axes in $B-D$ are in logarithmic scale.

on (1) the results of the linear mixed models described in the previous section whenever these showed significant relationships (i.e., for the scale parameter of migratory distance distributions, $\mu_{\text {mig }}$ and the scale parameter of the seed retention time distributions, $\mu_{\mathrm{GRT}}$; see "Results") and (2) the relationship reported by Alerstam et al. (2007) for bird flight speed. Parameters not related to bird mass or with unknown relationships were kept fixed in all the model runs.

\section{Results}

\section{Model Results}

The seed dispersal curves generated by waterbird movements during the migration season were highly leptokurtic and skewed toward local-scale dispersal (dispersal distances of $100 \mathrm{~km}$ or shorter; fig. 2), but a high proportion (95\%) of the estimated dispersal curves had a tail of LDD events (dispersal distances longer than $100 \mathrm{~km}$ ). The den- 
A

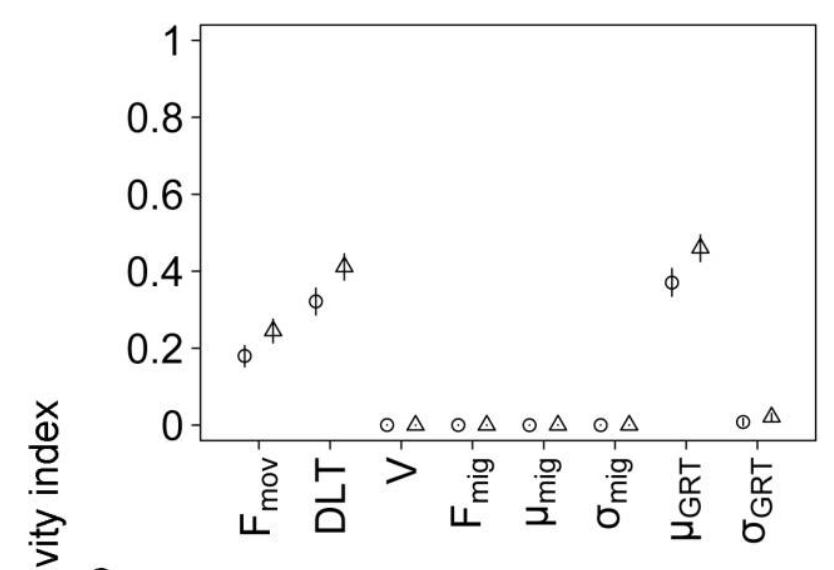

B

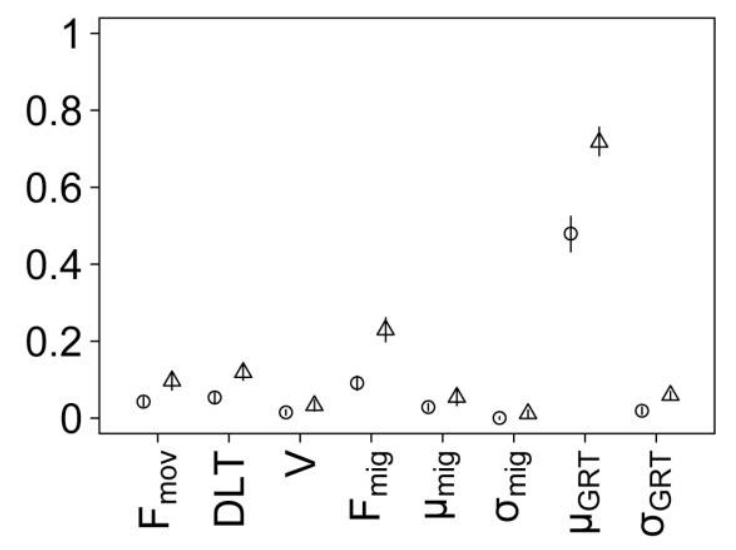

D

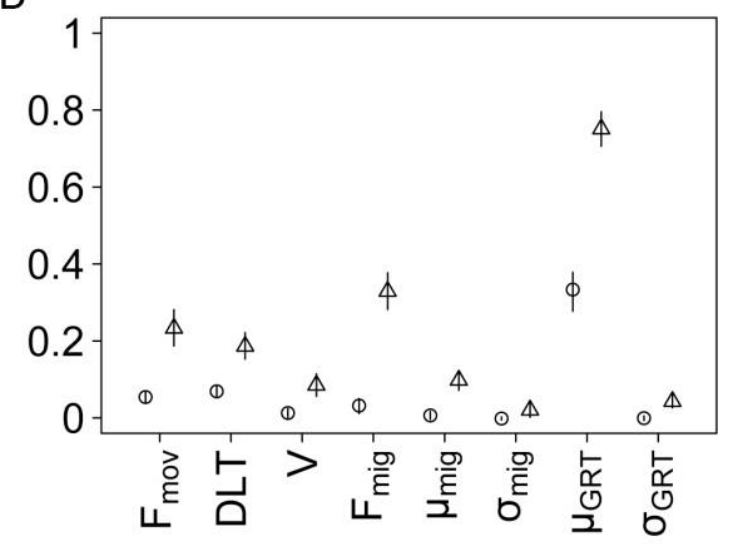

Model parameters

Figure 3: Results from the sensitivity analysis for the various model outputs: frequency of transported seeds $(A)$, frequency of long-distance dispersal events $\left(F_{\mathrm{LDD}} ; B\right)$, median dispersal distance $(C)$, and the $99 \%$ quantile of the dispersal distances $\left(Q_{99} ; D\right)$. Plots show the firstorder (circles \pm confidence interval $[\mathrm{CI}]$ ) and total (triangles $\pm \mathrm{CI}$ ) sensitivity index for the different parameters.

sity of the tail varied with the frequency of LDD events $\left(F_{\mathrm{LDD}}\right)$, which varied between $0 \%$ and $3.5 \%$ (median $=$ $0.1 \%$; fig. $2 A, 2 B$ ). Marked differences in the density of the curve tails resulted in considerable differences in the median dispersal distances, which varied from 6 to $81 \mathrm{~km}$ $($ median $=18 \mathrm{~km}$; fig. $2 C)$. Seed dispersal could reach extremely long distances (up to $3,000 \mathrm{~km}$ ), although the totality of the dispersal curves had $99 \%$ quantiles that did not rise above $518 \mathrm{~km}$ (fig. 2D). In fact, for half of the estimated dispersal curves, the $99 \%$ quantile never rose above $19 \mathrm{~km}$ (fig. 2D).

\section{Sensitivity Analysis}

Parameter variation had a strong effect on estimated dispersal patterns. However, the relative importance of each parameter varied for the different output variables (fig. 3).
The FED, which ranged from $0 \%$ to approximately $8 \%$, was mainly affected by the birds' probability of moving $\left(F_{\text {mov }}\right)$ and DLT, as well as the scale of seed retention times $\left(\mu_{\mathrm{GRT}}\right)$. Interactions between parameters showed weak effects on FED (fig. 3A). FED increased, on average, 4.5fold (from $1.2 \%$ to $5.3 \%$ ) with increasing $\mu_{\mathrm{GRT}}$ (which ranged from -0.01 to 2.41 , i.e., median seed retention times that ranged from approximately 1 to $11 \mathrm{~h}$ ), 2.8 -fold with increasing $F_{\text {mov }}$ (which ranged from $3 \%$ to $8 \%$ ), and 1.5 -fold with decreasing averaged DLT after seed ingestion. The latter effect means that birds that tend to depart immediately after seed ingestion (behavior 1) disperse, on average, 1.5 times more seeds than those that tend to wait (behavior 2; random times of departure showed intermediate FEDs).

The frequency of long-distance dispersal $\left(F_{\mathrm{LDD}}\right)$ was mainly affected by the scale of seed retention times $\left(\mu_{\mathrm{GRT}}\right)$ 

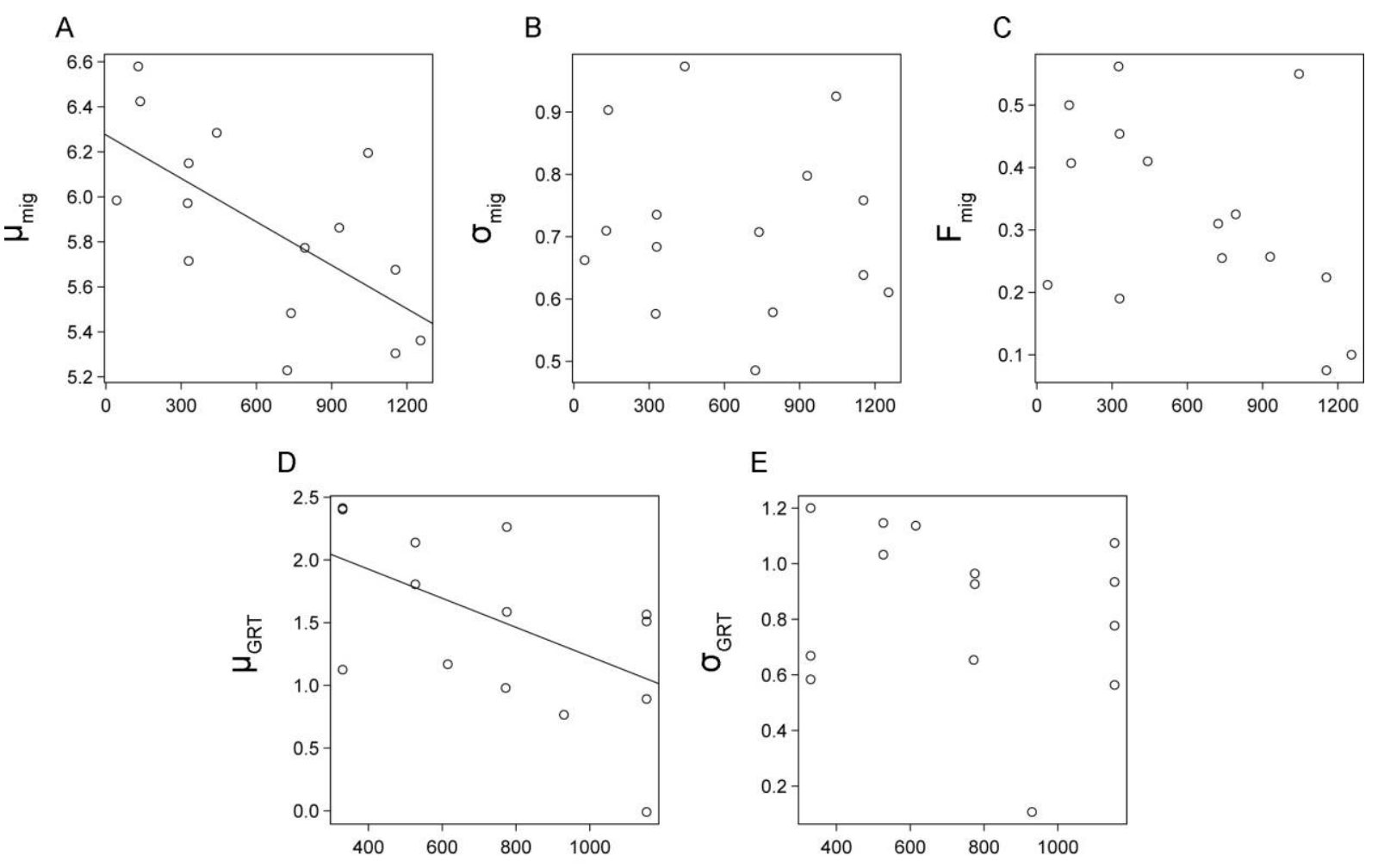

Bird species mass $(\mathrm{g})$

Figure 4: Effect of interspecific variation in bird body mass on the parameters describing bird migratory movement $(A-C)$ and seed gut retention time $(D, E)$. Parameters are dimensionless because they correspond either to the natural logarithm of distance $(\mathrm{km} ; A, B)$ and time $(\mathrm{h} ; D, E)$ or to a frequency $(C)$. Note that the scale of the horizontal axes of migratory movement $(A-C)$ and seed retention time $(D, E)$ differ.

and the frequency of migratory movements $\left(F_{\text {mig }}\right.$; fig. $\left.3 B\right)$, increasing, on average, 88 -fold (from $0.008 \%$ to $0.7 \%$ ) with increasing $\mu_{\mathrm{GRT}}$ (from -0.01 to 2.41 , as above) but only 2.7 -fold (from $0.09 \%$ to $0.25 \%$ ) with increasing $F_{\text {mig }}$ (which ranged from 0.08 to 0.56 ). Modest interaction effects among parameters also affected $F_{\mathrm{LDD}}$.

The median dispersal distance was strongly affected by $\mu_{\mathrm{GRT}}$ and $F_{\text {mig }}$ but was also affected by the scale of migratory distances $\left(\mu_{\text {mig; }}\right.$; the median varied between 187 and 720 $\mathrm{km}$ ) and the shape of seed retention time ( $\sigma_{\mathrm{GRT}}$; fig. $3 C$ ). Likewise, $\mu_{\mathrm{GRT}}$ and $F_{\mathrm{mig}}$ were the most influential parameters for the $99 \%$ quantile, which was also influenced by strong interactions in all parameters (fig. $3 D$ ).

\section{Effect of Bird Body Mass on Migratory Movement and Seed Retention Time}

Bird mass was negatively correlated with the scale parameters of both migratory distance $\left(\mu_{\text {mig }} ; \chi^{2}=4.79, \mathrm{df}=\right.$ $1, P=.03$; fig. $4 A)$ and seed retention times $\left(\mu_{\mathrm{GRT}}\right.$; $\chi^{2}=5.42, \mathrm{df}=1, P=.02$; fig. $\left.4 D\right)$. However, its effect on the frequency of migratory movements $\left(F_{\mathrm{mig}} ; \chi^{2}=\right.$ 2.58, $\mathrm{df}=1, P=.11$; fig. $4 C)$ and the shape parameter of both migratory distance $\left(\sigma_{\text {mig }} ; \chi^{2}=0.14, \mathrm{df}=1\right.$, $P=.71$; fig. $4 B)$ and seed retention times $\left(\sigma_{\mathrm{GRT}} ; \chi^{2}=\right.$ 0.60 , df $=1, P=.44$; fig. $4 E$ ) were not significant.

When the scale parameter $(\mu)$ of the lognormal distributions fitted to the migratory distances and seed retention times was substituted by the empirical log-transformed median, the relationships were also significant and very similar to those described for $\mu$ (in the case of retention times, sampling times were smoothed to make the data more continuous; data not shown).

\section{Effect of Bird Mass on Seed Dispersal Patterns}

Owing to its positive effects on the scale parameters of both migratory movement distance and seed retention time $\left(\mu_{\text {mig }}\right.$ and $\left.\mu_{\mathrm{GRT}}\right)$, discussed in the previous section, and its positive effect on flight speed, bird mass had strong indirect effects on the variables of frequency and distance of effective dispersal (fig. 5). Both of these latter variables 


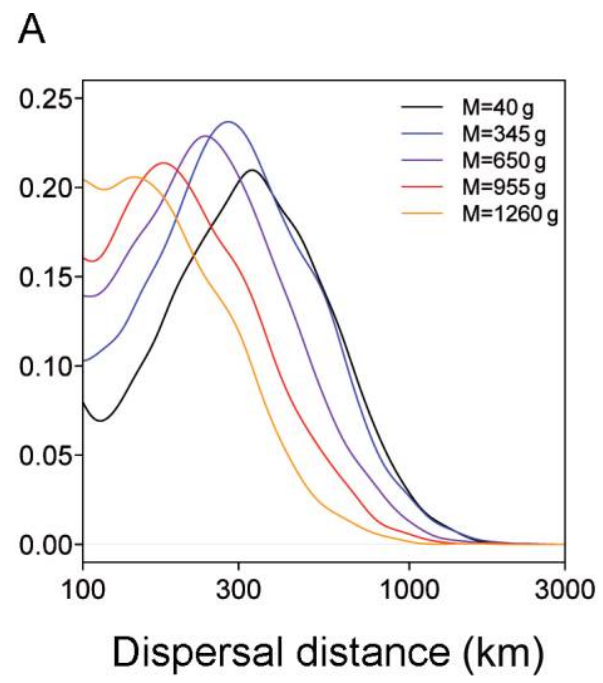

B

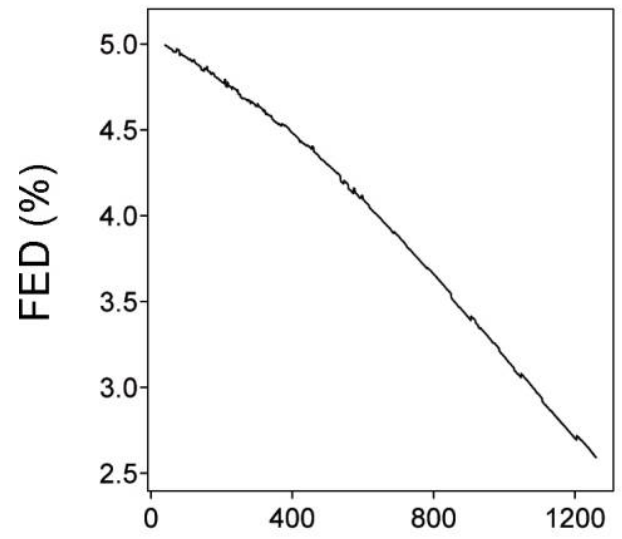

D

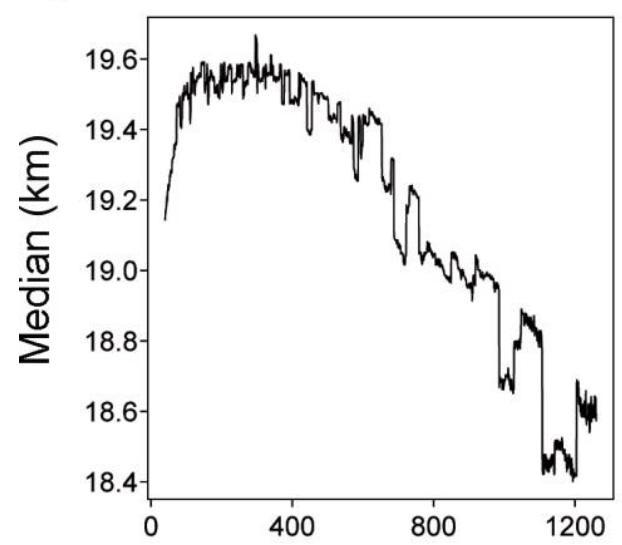

C

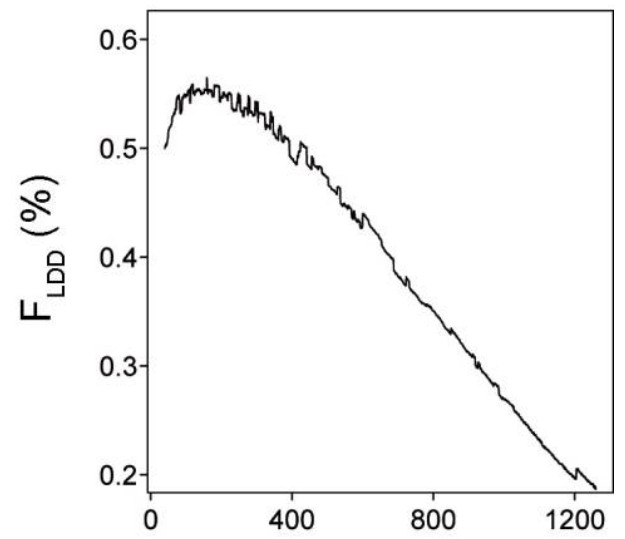

$\mathrm{E}$

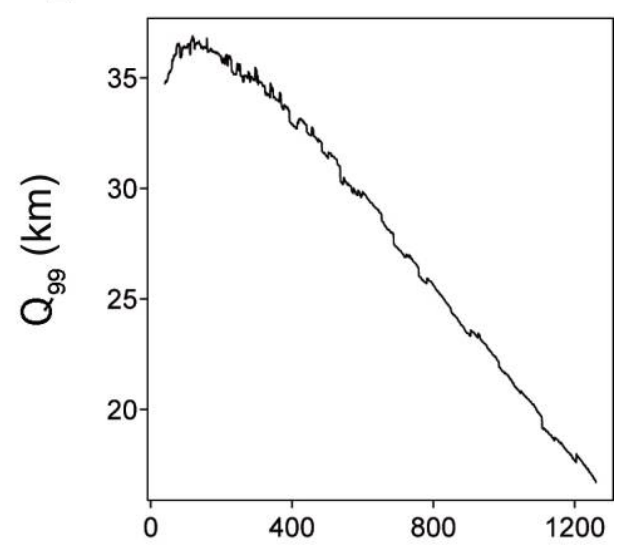

Bird mass (g)

Figure 5: Effect of interspecific variation in bird body mass on seed dispersal patterns. Dispersal patterns are represented by the seed dispersal curves generated by five values of bird body mass $(A)$, the frequency of effective dispersal (FED; $B)$, the frequency of long-distance dispersal events $\left(F_{\mathrm{LDD}} ; C\right)$, the median dispersal distance $(D)$, and the $99 \%$ quantile of the dispersal distances $\left(Q_{99} ; E\right)$. 
decreased with increased bird mass, owing largely to the greater influence that the two former parameters (migratory movement distance and seed retention time), compared with the latter (flight speed), have on dispersal patterns (as shown in "Sensitivity Analysis") and to the fact that flight speed varies little with body mass.

\section{Discussion}

By means of a simple mechanistic model, this article tracks down the processes involved in the generation of successful propagule LDD by migratory birds. Two basic processes formed the structure of the model: bird migratory behavior and seed GRT. Our results show that the size of the vector bird scales to two key model parameters (bird migratory distance and seed retention time) that strongly affect the seed dispersal patterns (dispersal frequency and distance). Because mechanistic models serve the purpose of understanding the system in a process-based fashion and are therefore suitable for generalization (Nathan and MullerLandau 2000; Cousens et al. 2010), bird size provides a straightforward measure by which the potential of bird species as propagule dispersers can be generalized. As our understanding of the dispersal processes increases, we should be able to assess the importance of many migratory bird vectors (waterbirds and land birds) by estimating the frequency and distance of propagule dispersal for the vectored organisms.

The importance and effectiveness of a vector species is largely determined by the frequency with which it transports propagules and deposits them into suitable habitat. According to our results, the FED depends mainly on the vector probability of moving, the time it takes to depart (DLT), and the scale of seed retention times $\left(\mu_{\mathrm{GRT}}\right)$. The only situation in which seed dispersal might not take place is when birds stop feeding well before departing (when DLT > GRT), particularly if propagules have short retention times. This may well be the case for some species of shorebirds, such as the bar-tailed godwit (Limosa lapponica) and the red knot (Calidris canutus), which have been shown to stop feeding from several hours to up to 1 or 2 days before departure to allow for physiological and/or morphological alterations (e.g., Piersma and Gill 1998). Indeed, while shorebirds feed on both seeds and propagules of several aquatic organisms (e.g., Green et al. 2005; Sánchez et al. 2006) and migrate over long distances, some species (notably, the longest migrants) might not be able to function as propagule dispersers because they atrophy their guts before departing for migratory flights (Piersma and Gill 1998).

Waterbirds dispersed a maximum of 3.5\% (the median was $0.1 \%$ ) of the defecated viable seeds over more than 100 $\mathrm{km}$ (note that if the model is to be parameterized for other bird groups, the LDD may need to be redefined according to the species' movement behavior). This frequency should be even lower when calculated on the basis of ingested (rather than defecated viable) propagules, since a considerable proportion of them fail to survive gut passage or germinate afterward. For example, only $8 \%-50 \%$ of propagules of aquatic organisms found in droppings of wild waterbirds collected in the field were still able to hatch or germinate (Figuerola and Green 2002; Green et al. 2008; Brochet et al. 2009a). The experimental data used in this study showed similar viability values for defecated propagules $(5 \%-52 \%)$; however, only $10 \%-42 \%$ of ingested propagules were retrieved (i.e., not broken or digested) after gut passage, suggesting that only $0.5 \%-22 \%$ of ingested propagules will be viable when defecated (Charalambidou et al. 2003b, 2005; Figuerola et al. 2010). On the basis of these numbers, the maximum frequency of LDD estimated by our model would represent $0.8 \%$ of the ingested propagules. This frequency might decrease further, since propagule viability tends to decrease with increasing GRT (Charalambidou et al. 2003b, 2005; but see Figuerola et al. 2010). More work is needed to investigate the characteristics of both the vector species and the ingested propagules mediating this effect. Although the odds seem to be against LDD, the large number of migratory birds (e.g., tens of millions of waterbirds in Europe; Delany and Scott 2006) may suffice to translate these low frequencies into a reasonably high number of events taking place every year.

In addition to the frequency, the distance of the dispersal events is crucial to assess the effectiveness and role of a given dispersal vector (Nathan et al. 2007). In the case of migratory birds that act as LDD vectors, the distance of propagule dispersal depends largely on the migratory strategy of the vector bird species and the resulting movement patterns (e.g., the distribution of migratory distances; Viana et al. 2012). Although migratory distance can be affected by such external factors as weather conditions and landscape features, it is largely a function of flight efficiency, particularly as it relates to such features as aerodynamics and flight performance (e.g., body mass, wing size and shape, and wing loading; Hedenström 2008). Body mass, in particular, affects the birds' flight speed (see Alerstam et al. 2007 for a discussion) and migratory distance (the scale parameter $\mu_{\text {mig }}$, as observed in our study). Given the opposite effects of body mass on flight speed (positive) and migratory distance (negative), we can expect a trade-off between flight speed and migratory distance mediated by bird size, which (according to our simulations) has a bearing on seed dispersal. For example, smaller waterbirds tend to fly longer distances at lower speeds, which should increase the probability of propagule defecation while still in flight. However, body mass negatively influenced seed retention times; therefore, the longer GRTs 
of smaller waterbirds should compensate (at least in part) for the longer time spent on their migratory flights. In contrast, larger waterbirds show shorter GRTs and tend to fly shorter distances at higher speeds. Overall, the contrasting influence that bird body size has on three different determinants of seed dispersal identified by our model (flight speed, migratory distance, and GRT) results in a trade-off between the efficiency of dispersal over moderate distances and the potential for LDD-with a shift from the former to the latter as bird body size decreases. We therefore suggest that different bird species contribute in different ways to propagule dispersal, even when transporting propagules of the same organism (i.e., they may show differential contributions to its total dispersal kernel, sensu Nathan 2007).

If potential migratory and other long-distance movements mediating seed LDD are not taken into account, the estimated dispersal kernel of many plant taxa belonging to different biomes might be truncated (i.e., the dispersal tails might be underestimated). Many migratory birds inhabiting different biogeographical regions are known to ingest seeds and potentially disperse them. The vast majority of migratory seed dispersers, such as frugivorous birds (e.g., Johnson et al. 1985), waterbirds (e.g., Brochet et al. 2009b), and many insectivorous migrants that also consume fruit during the migration season (compensatory frugivory; Eggers 2000), live in temperate and boreal regions. However, tropical regions also harbor migratory frugivorous species-some make altitudinal migrations (e.g., Levey and Stiles 1992), and others make latitudinal migrations between temperate and tropical regions (Chesser and Levey 1998) - that can therefore act as vectors of seed LDD. Temperate-tropical migrants may, however, show limited potential to mediate effective longdistance seed dispersal, as plants might not be able to establish in such different habitats. On the contrary, aquatic environments are more homogeneous and therefore are more amenable to successful LDD events, even across different biomes. Because LDD influences population dynamics and distribution patterns at higher scales (other than local; Fragoso et al. 2003) as well as the range expansion rate of species (Higgins and Richardson 1999), investigating the potential of migratory birds to mediate long-distance seed dispersal in different biomes can help to explain the biogeographical patterns of many plant species.

The model presented here might be adapted and parameterized to study the dispersal of terrestrial plants by land birds. It is important to note, however, that the allometric relationships found in this study might vary among different bird taxa. For example, the body size of passerine species was shown to have a positive effect on GRT (Karasov 1990). A positive relationship might even be frequent because propagules passing through longer guts, such as those of larger birds, should take more time. However, other factors might affect seed retention time. For example, bird size is also related to gizzard size in a positive way (as observed by performing a Pearson's correlation test with data provided in Herrera 1984; data not shown), and according to Figuerola et al. (2002) larger gizzards destroy larger numbers of seeds (at least in waterfowl). This might mean that only the seeds that spend less time in the gizzard can survive, which could result in shorter retention times (as the bird body mass increases).

Further model developments should also address the effects of other parameters on LDD. For instance, for both waterbirds and land birds the effect of vector activity and time activity budgets on propagule retention time may be important. For example, the swimming activity of ducks was shown to reduce propagule retention time compared with that of resting ducks (van Leeuwen et al. 2012). Propagule characteristics, such as seed size (Soons et al. 2008), may also affect LDD. This model could also be applied to pathogen dispersal if seed retention time is replaced by the duration of infectious periods in birds (e.g., 1-8 days for H5N1 avian influenza; Gaidet et al. 2010). However, the potential effects of infection on the migration capacity of birds (e.g., van Gils et al. 2007) should be accounted for in the model.

The simple mechanistic approach presented here provides a framework for LDD hypothesis testing in relation to colonization success, connectivity, or invasion spread. For example, assessing the bird potential to disperse seeds of plants with known range expansion rates would allow researchers to compare such rates with the estimated frequency and distance range of seed LDD. The calibration and validation of such models, in both terrestrial and aquatic habitats, would be of key importance to explain biogeographic patterns, predict future range shifts (e.g., in response to climate change or as a result of biological invasions), and/or infer the impact of altered landscape dynamics (e.g., in response to habitat fragmentation or wetland loss). This work is a first step toward the development of a general framework for modeling, understanding, and forecasting biotic transport by migratory birds.

\section{Acknowledgments}

We are grateful for the banding and recovery data freely provided by the Bird Banding Laboratory (US Geological Survey) and purchased from EURING (European Union for Bird Banding), and we feel indebted to all the voluntary banders and national schemes that generated them in the first place. C. du Feu and W. Fiedler greatly facilitated the release of EURING data for research purposes. We also 
thank M. A. Rodríguez-Gironés for mathematical advice $\rightarrow$ Eggers, S. 2000. Compensatory frugivory in migratory Sylvia warand two anonymous reviewers for helping us clarify the blers: geographical responses to season length. Journal of Avian Biology 31:63-74. modeling approach. The study was funded by ESFEURODIVERSITY project BIOPOOL through project CGL2006-05085/BOS cofinanced by ERDF (European Regional Development Funds). D.S.V. was supported by Fundação para a Ciência e Tecnologia grant SFRH/BD $48091 / 2008$, cofinanced by the European Social Fund, and J.F. was supported by Spanish Ministry of Science project CGL2009-11445 and Junta de Andalucia project RNM04744.

Figuerola, J., I. Charalambidou, L. Santamaría, and A. Green. 2010. Internal dispersal of seeds by waterfowl: effect of seed size on gut passage time and germination patterns. Naturwissenschaften 97 : $555-565$.

Figuerola, J., and A. J. Green. 2002. Dispersal of aquatic organisms by waterbirds: a review of past research and priorities for future studies. Freshwater Biology 47:483-494.

Figuerola, J., A. J. Green, and T. C. Michot. 2005. Invertebrate eggs can fly: evidence of waterfowl-mediated gene flow in aquatic invertebrates. American Naturalist 165:274-280.

$\rightarrow$ Figuerola, J., A. J. Green, and L. Santamaría. 2002. Comparative dispersal effectiveness of wigeongrass seeds by waterfowl wintering in south-west Spain: quantitative and qualitative aspects. Journal of Ecology 90:989-1001.

\section{Literature Cited}

$\rightarrow$ Alerstam, T., M. Rosén, J. Bäckman, P. G. P. Ericson, and O. Hellgren 2007. Flight speeds among bird species: allometric and phylogenetic effects. PLoS Biology 5:e197.

$\rightarrow$ Bohrer, G., R. Nathan, and S. Volis. 2005. Effects of long-distancr dispersal for metapopulation survival and genetic structure at ecological time and spatial scales. Journal of Ecology 93:1029-1040.

$\rightarrow$ Brochet, A.-L., M. Guillemain, H. Fritz, M. Gauthier-Clerc, and A J. Green. 2009a. Plant dispersal by teal Anas crecca in the Camargue: duck guts are more important than their feet. Freshwater Biology 55:1262-1273.

$\rightarrow-2009 \mathrm{~b}$. The role of migratory ducks in the long-distance dispersal of native plants and the spread of exotic plants in Europe Ecography 32:919-928.

$\rightarrow$ Bruderer, B., and A. Boldt. 2001. Flight characteristics of birds. I. Radar measurements of speeds. Ibis 143:178-204.

$\rightarrow$ Charalambidou, I., and L. Santamaría. 2005. Field evidence for the potential of waterbirds as dispersers of aquatic organisms. Wetlands 25:252-258.

$\rightarrow$ Charalambidou, I., L. Santamaría, and J. Figuerola. 2003a. How far can the freshwater bryozoan Cristatella mucedo disperse in duck guts? Archiv für Hydrobiologie 157:547-554.

$\rightarrow$ Charalambidou, I., L. Santamaría, C. Jansen, and B. A. Nolet. 2005. Digestive plasticity in mallard ducks modulates dispersal probabilities of aquatic plants and crustaceans. Functional Ecology 19: 513-519.

$\rightarrow$ Charalambidou, I., L. Santamaría, and O. Langevoord. 2003b. Effec of ingestion by five avian dispersers on the retention time, retrieval and germination of Ruppia maritima seeds. Functional Ecology 17:747-753.

$\rightarrow$ Chesser, R. T., and D. J. Levey. 1998. Austral migrants and the evolution of migration in New World birds: diet, habitat, and mi gration revisited. American Naturalist 152:311-319.

$\rightarrow$ Clark, J. S. 1998. Why trees migrate so fast: confronting theory with dispersal biology and the paleorecord. American Naturalist 152. 204-224.

$\rightarrow$ Clausen, P., B. A. Nolet, A. D. Fox, and M. Klaassen. 2002. Longdistance endozoochorous dispersal of submerged macrophyte seeds by migratory waterbirds in northern Europe-a critical review of possibilities and limitations. Acta Oecologica 23:191-203.

$\rightarrow$ Cousens, R. D., J. Hill, K. French, and I. D. Bishop. 2010. Towards better prediction of seed dispersal by animals. Functional Ecology 24:1163-1170.

Delany, S., and D. Scott. 2006. Waterbird population estimates. Wetlands International, Wageningen.

- 2003. Passive internal transport of aquatic organisms by waterfowl in Doñana, south-west Spain. Global Ecology and Biogeography 12:427-436.

Fragoso, J. M. V., K. M. Silvius, and J. A. Correa. 2003. Long-distance seed dispersal by tapirs increases seed survival and aggregates tropical trees. Ecology 84:1998-2006.

Gaidet, N., J. Cappelle, J. Y. Takekawa, D. J. Prosser, S. A. Iverson, D. C. Douglas, W. M. Perry, et al. 2010. Potential spread of highly pathogenic avian influenza $\mathrm{H} 5 \mathrm{~N} 1$ by wildfowl: dispersal ranges and rates determined from large-scale satellite telemetry. Journal of Applied Ecology 47:1147-1157.

Gillespie, R. G., B. G. Baldwin, J. M. Waters, C. I. Fraser, R. Nikula, and G. K. Roderick. 2012. Long-distance dispersal: a framework for hypothesis testing. Trends in Ecology and Evolution 27:47-56.

Green, A. J., K. M. Jenkins, D. Bell, P. J. Morris, and R. T. Kingsford. 2008. The potential role of waterbirds in dispersing invertebrates and plants in arid Australia. Freshwater Biology 53:380-392.

$\rightarrow$ Green, A. J., M. I. Sanchez, F. Amat, J. Figuerola, F. Hontoria, O. Ruiz, and F. Hortas. 2005. Dispersal of invasive and native brine shrimps Artemia (Anostraca) via waterbirds. Limnology and Oceanography 50:737-742.

Hedenström, A. 2008. Adaptations to migration in birds: behavioral strategies, morphology and scaling effects. Philosophical Transactions of the Royal Society B: Biological Sciences 363:287-299.

$\rightarrow$ Hein, A. M., C. Hou, and J. F. Gillooly. 2012. Energetic and biomechanical constraints on animal migration distance. Ecology Letters 15:104-110.

$\rightarrow$ Herrera, C. M. 1984. Adaptation to frugivory of Mediterranean avian seed dispersers. Ecology 65:609-617.

$\rightarrow$ Higgins, S. I., and D. M. Richardson. 1999. Predicting plant migration rates in a changing world: the role of long-distance dispersal. American Naturalist 153:464-475.

Johnson, R. A., M. F. Willson, J. N. Thompson, and R. I. Bertin. 1985. Nutritional values of wild fruits and consumption by migrant frugivorous birds. Ecology 66:819-827.

Karasov, W. H. 1990. Digestion in birds: chemical and physiological determinants and ecological implications. Studies in Avian Biology 13:391-415.

Lehnen, S. E., and D. G. Krementz. 2007. The influence of body condition on the stopover ecology of least sandpipers in the Lower Mississippi Alluvial Valley during fall migration. Avian Conservation and Ecology 2:9.

$\rightarrow$ Levey, D. J., and F. G. Stiles. 1992. Evolutionary precursors of long- 
distance migration: resource availability and movement patterns in Neotropical landbirds. American Naturalist 140:447-476.

$\rightarrow$ Levey, D. J., J. J. Tewksbury, and B. M. Bolker. 2008. Modelling longdistance seed dispersal in heterogeneous landscapes. Journal of Ecology 96:599-608.

$\rightarrow$ Levine, J. M., and D. J. Murrell. 2003. The community-level con $\rightarrow$ sequences of seed dispersal patterns. Annual Review of Ecology, Evolution, and Systematics 34:549-574.

$\rightarrow$ Lislevand, T., J. Figuerola, and T. Székely. 2007. Avian body sizes ir relation to fecundity, mating system, display behavior and resource sharing. Ecology 88:1605.

$\rightarrow$ Miller, M. R., J. Y. Takekawa, J. P. Fleskes, D. L. Orthmeyer, M. L. Casazza, and W. M. Perry. 2005. Spring migration of northerr $\rightarrow$ pintails from California's Central Valley wintering area tracked with satellite telemetry: routes, timing, and destinations. Canadian Journal of Zoology 83:1314-1332.

Nathan, R. 2007. Total dispersal kernels and the evaluation of diversity and similarity in complex dispersal systems. Pages 252-276 in A. J. Dennis, E. W. Schupp, R. J. Green, and D. A. Westcott $\rightarrow$ eds. Seed dispersal: theory and its application in a changing world. CAB International, Wallingford.

$\rightarrow$ Nathan, R., and H. C. Muller-Landau. 2000. Spatial patterns of seer $\rightarrow$ dispersal, their determinants and consequences for recruitment. Trends in Ecology and Evolution 15:278-285.

$\rightarrow$ Nathan, R., G. Perry, J. T. Cronin, A. E. Strand, and M. L. Cain. 2003. Methods for estimating long-distance dispersal. Oikos 103 261-273.

$\rightarrow$ Nathan, R., F. M. Schurr, O. Spiegel, O. Steinitz, A. Trakhtenbrot, and A. Tsoar. 2008. Mechanisms of long-distance seed dispersal. Trends in Ecology and Evolution 23:638-647.

Newton, I. 2008. The migration ecology of birds. Academic Press, Oxford.

$\rightarrow$ O’Neal, B. J., J. D. Stafford, and R. P. Larkin. 2012. Stopover duratior of fall-migrating dabbling ducks. Journal of Wildlife Management 76:285-293.

$\rightarrow$ Owen, R. B. 1968. Premigratory behavior and orientation in bluewinged teal (Anas discors). Auk 85:617-632.

$\rightarrow$ Piersma, T., and R. E. Gill Jr. 1998. Guts don't fly: small digestive organs in obese bar-tailed godwits. Auk 115:196-203.

$\rightarrow$ Popp, M., V. Mirré, and C. Brochmann. 2011. A single MidPleistocene long-distance dispersal by a bird can explain the extreme bipolar disjunction in crowberries (Empetrum). Proceedings of the National Academy of Sciences of the USA 108:6520-6525.

$\rightarrow$ Rawsthorne, J., D. A. Roshier, and S. R. Murphy. 2009. A simple parametric method for reducing sample sizes in gut passage time trials. Ecology 90:2328-2331.

R Development Core Team. 2009. R: a language and environment for statistical computing. R Foundation for Statistical Computing, Vienna. http://www.R-project.org.

Saltelli, A. 2002. Making best use of model evaluations to compute sensitivity indices. Computer Physics Communications 145:280 297.

Sánchez, M. I., A. J. Green, and E. M. Castellanos. 2006. Internal transport of seeds by migratory shorebirds in the Odiel marshes, south-west Spain: consequences for long-distance dispersal. Journal of Avian Biology 37:201-206.

Santamaría, L. 2002. Why are most aquatic plants widely distributed? dispersal, clonal growth and small-scale heterogeneity in a stressful environment. Acta Oecologica 23:137-154.

$\rightarrow$ Soons, M. B., C. van der Vlugt, B. van Lith, G. W. Heil, and M. Klaassen. 2008. Small seed size increases the potential for dispersal of wetland plants by ducks. Journal of Ecology 96:619-627.

Trakhtenbrot, A., R. Nathan, G. Perry, and D. M. Richardson. 2005. The importance of long-distance dispersal in biodiversity conservation. Diversity and Distributions 11:173-181.

van Gils, J. A., V. J. Munster, R. Radersma, D. Liefhebber, R. A. M. Fouchier, and M. Klaassen. 2007. Hampered foraging and migratory performance in swans infected with low-pathogenic avian influenza A virus. PLoS ONE 2:e184.

van Leeuwen, C., M. Tollenaar, and M. Klaassen. 2012. Vector activity and propagule size affect dispersal potential by vertebrates. Oecologia 170:101-109.

Viana, D. S., L. Santamaría, T. C. Michot, and J. Figuerola. 2012. Migratory strategies of waterbirds shape the continental-scale dispersal of aquatic organisms. Ecography, doi:10.1111/j.1600 $-0587.2012 .07588 . x$.

Welham, C. V. J. 1994. Flight speeds of migrating birds: a test of maximum range speed predictions from three aerodynamic equations. Behavioral Ecology 5:1-8.

Wernham, C. V., M. P. Toms, J. H. Marchant, J. A. Clarck, G. M. Siriwardena, and S. R. Baillie. 2002. The migration atlas: movements of the birds of Britain and Ireland. Poyser, London.

Zwarts, L., B. J. Ens, M. Kersten, and T. Piersma. 1990. Moult, mass and flight range of shorebirds ready to take off for long-distance migrations. Ardea 78:339-364.

Associate Editor: Robert Dudley Editor: Judith L. Bronstein 